\title{
Uso de drones y sensores remotos para el monitoreo de laderas: una revisión
}

\author{
Using Drones and Remote Sensing for Slope Monitoring: a Review
}

Luis Fernando Alonso Gómez ${ }^{1}$ Saieth Baudilio Chaves Pabón ${ }^{2}$

\section{OPEN ACCESS}

\section{(c) $(1) \Theta$}

Copyright:

(02021. La revista Ingenierías USBmed proporciona acceso abierto a todos sus contenidos bajo los términos de la licencia creative commons Atribución no comercial SinDerivar 4.0 Internacional (CC BY-NC-ND 4.0)

Tipo de artículo: Revisión. Recibido: 03-12-2020.

Revisado: 13-04-2021.

Aprobado: 25-05-2021.

Doi: $10.21500 / 20275846.5158$

\section{Referenciar así:}

L. F. Alonso Gómez y S. B. Chavez Pabón, "Uso de drones y sensores remotos para el monitoreo de laderas: una revisión," Ingenierías USBMed, vol. 12, n. ${ }^{\circ}$ 2, pp. 65-73, 2021.

\section{Disponibilidad de datos:}

todos los datos relevantes están dentro del artículo, así como los archivos de soporte de información.

\section{Conflicto de intereses:}

los autores han declarado que no hay conflicto de intereses.

Editor: Andrés Felipe Hernández. Universidad de San Buenaventura, Medellín, Colombia.

\author{
${ }^{1}$ Estudiante del Programa de Ingeniería Civil. Facultad de Estudios a Distan- \\ cia. Universidad Militar Nueva Granada, Bogotá, Colombia. Email: d7303533@ \\ unimilitar.edu.co \\ ${ }^{2}$ Ingeniero Civil. PhD en Ingeniería Geodésica y Cartografía. Profesor Asistente \\ del Programa de Ingeniería Civil. Facultad de Estudios a Distancia. Universidad \\ Militar Nueva Granada, Bogotá, Colombia. Email: saieth.chaves@unimilitar.edu.co
}

Resumen. Los deslizamientos de tierra son uno de los peligros y riesgos más costoso y fatales para cualquier infraestructura vial, que frecuentemente amenazan e influyen en la situación socioeconómica, principalmente, de los países en vía de desarrollo. Frecuentemente, los estudios de deslizamiento se hacen de manera directa, in situ, siendo muchos de ellos costosos y un reto dada la dificultad que se pueden presentar al acceder a las zonas de estudio. Los datos de los sensores remotos se pueden usar en el monitoreo de deslizamientos de tierra, mapeo, simulación, predicción y evaluación de peligros y otras investigaciones. El presente artículo presenta una revisión bibliográfica de estudios relacionados con el uso de drones y sensores remotos para el seguimiento y monitoreo de laderas, de una manera descriptiva y argumentativa, a través de información seleccionada se puedan afrontar futuros estudios más detallados, que permitan tener en cuenta el monitoreo de deslizamientos de tierra, técnicas de sensores remotos, drones y la aplicación global de este conjunto de nuevas tecnologías.

Palabras Clave. Sensores remotos; drones; deslizamientos de tierra; detección; peligros naturales.

Abstract. Landslides are one of the most costly and fatal hazards and risks to any road infrastructure, often threatening and influencing the socio-economic situation mainly in developing countries. Landslide studies are often carried out directly in situ, many of them being costly and challenging given the difficulty of accessing the study areas. Remote sensing data can be used in landslide monitoring, mapping, simulation, hazard prediction and assessment and other research. This article presents a literature review of studies related to the use of drones and remote sensing for slope tracking and monitoring, in a descriptive and argumentative way, through selected information, more detailed future studies can be addressed, allowing to consider landslide monitoring, remote sensing techniques, drones and the global application of this set of new technologies.

Keywords. Remote Sensing; Drones; Landslides; Detection; Natural Hazards. 


\section{Introducción}

El monitoreo o seguimiento de laderas debido a posibles deslizamientos de tierra, actualmente hace surgir la necesidad de conocer el desarrollo de nuevas tecnologías que permitan monitorear estos probables eventos [1]. A través de los años, el desarrollo de la fotogrametría y sus aplicaciones, además de la modernización de las técnicas de geoposicionamiento, han abierto las puertas para el avance en el estudio de nuevas metodologías de análisis espacial que pueden permitir integrar información y modelos de evaluación más eficientes [2]

Por otra parte, se tienen los deslizamientos de tierra y fenómenos de remoción de masas en laderas, estabilizadas y no estabilizadas, que tienen un impacto significativo a nivel mundial [3], [4]. En comparación con otros peligros naturales, estos fenómenos naturales son unos de los peligros geológicos más costosos y fatales en términos de vidas humanas, que amenazan e influyen en las condiciones socioeconómicas de muchos países a nivel mundial [5], [6].

Un deslizamiento de tierra en laderas puede ser provocado por varios fenómenos naturales (por ejemplo, terremotos, lluvias torrenciales, tsunamis e inundaciones) y perturbaciones humanas (por ejemplo, deforestación, desarrollo de infraestructura por cortes de pendientes y presencia de cavidades subterráneas históricas) [7], [8]. En el caso de Estados Unidos, los deslizamientos de tierra causan aproximadamente $\$ 3.5$ mil millones en daños y matan entre 25 y 50 personas cada año [9]. Además, solo en 2014, Nepal tuvo un deslizamiento de tierra en laderas en donde se contabilizó una gran pérdida de ganado, muy superior a las pérdidas de seres humanos, adicionalmente los daños en la infraestructura vial fueron incontables, perjudicando de manera irreversible a la economía del país [10]. Las fallas de las pendientes también provocan una importante sedimentación en los arroyos y lagos, lo que representó además una de las principales causas de inundaciones.

Los deslizamientos de tierra en laderas son comunes principalmente en las regiones montañosas, sobre todo si se tiene una pendiente inestable o se vuelve inestable debido a fuerzas impulsoras externas [11]. El peligro de deslizamiento de tierra en laderas se puede clasificar en alto, moderado y bajo en función del volumen, la duración, el posible efecto en términos de distancia, área y velocidad a la que falla la pendiente. Dado que los deslizamientos de tierra en laderas pueden afectar negativamente las vidas humanas y las propiedades, es esencial monitorear, detectar, mapear y realizar análisis de peligros para reducir el impacto de su peligro y salvar vidas humanas, propiedades y el medio ambiente. Los mapas de susceptibilidad a los deslizamientos de tierra en laderas se pueden desarrollar para las regiones propensas a los deslizamientos de tierra combinando todos los factores predisponen- tes potenciales que causan un deslizamiento de tierra en laderas.

Los Drones o VANT (Vehículos Aéreos No Tripulados) se utilizan cada vez más en actividades de investigación y en diferentes campos de aplicación, como la producción de cartografía [12], [13], supervisión de obras civiles [14], seguimiento de la agricultura [15]-[17], identificación de posibles zonas de riesgo [18], [19], manejo de desastres [20], [21], se han demostrado otros ejemplos de aplicaciones para el monitoreo de incendios forestales, como en el estudio de Zarco-Tejada y Berni [22] quienes utilizaron un UAV de ala fija con sensores térmicos e hiperespectrales. También se han reportado experimentos para clasificación de árboles [23], cálculo del Índice de Vegetación de Diferencia Normalizada [24] y monitoreo de temperaturas de arroyos [25]. Lo anterior muestra la versatilidad del uso de este tipo de tecnologías y su creciente aplicación en todos los renglones de la economía de un país, ofreciendo soluciones efectivas, de bajo costo, y de gran impacto.

En este documento se pretende analizar el uso de sensores remotos en trabajos de seguimiento y monitoreo de deslizamientos de tierra en laderas, sus técnicas y aplicaciones en estudios efectuados a nivel mundial.

\section{Metodología}

Esta investigación proyecta la realización del estado del conocimiento sobre el uso de drones y sensores remotos para el seguimiento y monitoreo de laderas estabilizadas y no estabilizadas. Se pretende generar bases de información apropiadas y acertadas que concierne información recolectada de documentos científicos basados en el manejo de drones y sensores remotos destinados al monitoreo de laderas o taludes. Dicho esto, se pretende reafirmar las técnicas que se han desarrollado actualmente para prevenir y mitigar deslizamientos o inestabilidades que puedan presentarse en la consecución de proyectos de Ingeniería Civil, habría que añadir que se busca reconocer una aproximación más real de cómo trabajan conjuntamente sensores remotos y drones en las laderas de los taludes, y la importancia de estos equipos en diversas actividades que demanda la Ingeniería Civil.

La metodología aplicada para esta investigación es de tipo documental [26], descriptiva del estado del conocimiento de investigaciones previas que aplican a casos de estudio sobre aplicaciones tomadas como ejemplo en el monitoreo de laderas de proyectos de Infraestructura Vial.

\section{Drones y el monitoreo de laderas}

Drones o UAV, por sus siglas en inglés "Unmanned Aerial Vehicles" o RPAS, por sus siglas en inglés "Remotely Pilot Aircraft System", o también conocidos como VANT, por sus siglas en español "Vehículos Aéreos 
No Tripulados". La facilidad de los drones para la consecución de imágenes aéreas y la probada prestación para capturar información en grandes zonas de estudio, adicionalmente a los avanzados desarrollos de la fotogrametría digital, han permitido almacenar y procesar abundante cantidad de datos cartográficos, geométricos, entre otros, que han permitido prevenir, responder y solucionar problemas de diseño, construcción y operación en proyectos de Ingeniería Civil. En este artículo se podrá encontrar un estudio del conocimiento enfocado al posible uso que se podría realizar en Colombia. En cuanto a proyectos de Ingeniería Civil, se han realizado adelantos significativos en proyectos de Infraestructura Vial como el uso de la topografía con Drones en la construcción de carreteras $4 \mathrm{G}$ y en el diseño de las nuevas carreteras 5G; igualmente, se podría mencionar su uso en la consecución de proyecto de Obras Hidráulicas ubicados sobre los ríos más importantes del país como el Magdalena y Cauca, donde el monitoreo de laderas, causes en ríos, deslaves, etc., son claves en la construcción de estas obras civiles.

La necesidad de hacer un seguimiento y un control efectivo de los deslizamientos de tierras en laderas de alta pendiente en infraestructura vial demanda de metodologías de fácil acceso con un costo sustancialmente bajo. Los Drones facilitan lo anterior debido a su capacidad de producir imágenes aéreas, de altas resoluciones en áreas de difícil acceso, que posteriormente serán procesados en ortomosaicos [27], los cuales serán georreferenciados y posicionados en el terreno a través de las observaciones satelitales [28], se pueden obtener MDT (Modelos Digitales del Terreno) que pueden ser creados en intervalos de tiempo determinados para su posterior comparación con el comportamiento de las laderas que posiblemente se puedan investigar o estudiar [29], [30]; así mismo, se podrán hacer correlaciones con los desplazamientos [31], para así poder obtener características geomorfológicas y climáticas [32].

De acuerdo con Giordan, Manconi, Remondino y Nex [19], se evidencia que el desarrollo reciente de UAV (Unmanned Aerial System) ha aumentado el número de soluciones técnicas que se pueden utilizar para monitorear y mapear los efectos de los peligros naturales. Para Berie y Burud [33], los UAV son generalmente más económicos y versátiles que las técnicas tradicionales de teledetección y, por lo tanto, se pueden considerar como una buena alternativa para la adquisición de imágenes y otros parámetros físicos antes, durante y después de un evento de amenaza natural. Este es un valor agregado importante, especialmente para investigaciones en áreas pequeñas.

En este contexto, los Drones o UAV ofrecen una resolución espacial sin precedentes y nuevas oportunidades de mapeo a escalas locales [34], donde la exploración cubre unos pocos kilómetros cuadrados y el uso de plataformas aéreas o satelitales podrían considerarse demasiado costosas. Además, Silvagni, Tonoli, Zenerino y Chiaberge [35] mencionan que los UAV presentan varias ventajas, que incluyen: (1) la capacidad de volar a bajas altitudes (menos de $150 \mathrm{~m}$ sobre el nivel del suelo), (2) la capacidad de llegar a ubicaciones remotas y capturar imágenes de alta resolución, (3) la capacidad para alojar diferentes sensores (cámaras, escáneres láser, sensores de navegación / inerciales, etc.), (4) la posibilidad de adquirir imágenes con diferentes ángulos, y (5) la flexibilidad de realizar operaciones de monitorización a pequeña, mediana y gran escala. También por estas razones, las plataformas UAV se utilizan comúnmente para la asistencia y gestión de emergencias. Por ejemplo, los UAV pueden proporcionar rápidamente información sobre edificios derrumbados después de un terremoto, como se puede evidenciar en distintos estudios [36]-[39].

Como se mencionó anteriormente, una de las características más útiles de los UAV es la posibilidad de adquirir bajo demanda un conjunto de datos de un área limitada [12], [13]. Esto es particularmente relevante cuando estos sistemas se adoptan para obtener información valiosa sobre un entorno particular y/o proceso geomorfológico. En los procesos que representan posibles riesgos geológicos, los efectos de la evolución de un proceso geológico y/o geomorfológico a menudo se pueden lograr mediante la comparación de información previa y posterior al evento en el área de estudio. En el pasado, este enfoque a menudo se apoyaba en el uso de LiDAR terrestres [40] o aerotransportados [41], pero la introducción de UAV representa hoy en día una alternativa valiosa para una adquisición multitemporal de conjuntos de datos que se pueden utilizar para el estudio de los peligros naturales, como efectivamente son los deslizamientos de tierras en laderas.

Para aplicaciones fotogramétricas, la carga útil de todo el sistema está compuesta por una cámara, un Sistema Global de Navegación por Satélite (GNSS) y una Unidad de Medición Inercial [42]. La cámara toma imágenes superpuestas mientras vuela sobre un área de estudio. Estas imágenes se pueden procesar a través de un flujo de trabajo fotogramétrico para obtener una nube de puntos o también conocido como Modelo Digital de Superficie, una ortofoto o un modelo 3D completo de la escena. Un dispositivo GNSS integrado permite georreferenciar estos datos. Sin embargo, en el contexto de los UAV de bajo costo, la precisión de dicho GNSS suele ser limitada [43], por lo tanto, los Puntos de Control Terrestre (GCP, por sus siglas en Inglés) suplementarios generalmente se adquieren en el área de estudio, para mantener la precisión de la orientación del bloque de imágenes y los productos cartográficos derivados, como las ortofotos, y para facilitar su integración con otros datos espaciales. Estos GCP deben seleccionarse cuidadosamente y estar bien distribuidos, 
y deben ser visibles en muchas imágenes, así como fácilmente identificables en las imágenes después de la adquisición y medibles con tecnología precisa, como GNSS de grado topográfico.

\section{Sensores remotos}

Nikolakopoulos et al. [44] expresan que un deslizamiento de tierra en laderas, activo se puede monitorear utilizando diferentes métodos como: mediciones geotécnicas clásicas donde se utiliza el inclinómetro, mediciones de levantamientos topográficos con estaciones totales o receptores de Sistemas Globales de Navegación por Satélite (GNSS, por sus siglas en inglés), Sistemas de detección y alcance de luz aerotransportada (LiDAR, por sus siglas en inglés), Escáneres Láser Terrestres (TLS, por sus siglas en inglés), técnicas fotogramétricas que utilizan fotografías aéreas o imágenes de satélite de alta resolución, Interferometría Diferencial utilizando imágenes de radar (DInSAR) y recientemente técnicas de visión por ordenador utilizando datos de UAV. [45] analizaron las ventajas y desventajas de utilizar estos diferentes métodos de detección.

Sin embargo, el método más reciente para el monitoreo de deslizamiento de tierra en laderas es la utilización de imágenes de ultra alta resolución capturadas desde UAV. Lucier, De Jong y Turner [46], además de Nikolakopoulos et al. [47] destacan que el uso de UAV con fines de investigación se ha vuelto posible y asequible debido a desarrollos tecnológicos como sistemas de piloto automático, cámaras de acción livianas, GNSS en miniatura, avances en fuselajes de fibra de carbono y el desarrollo simultáneo de nuevas metodologías de procesamiento basadas en visión por computadora como la Structure from Motion. Con la Structure from Motion se pueden obtener fotografías del objeto de interés con superposición suficiente (por ejemplo, 80-90\%) desde múltiples posiciones y / o ángulos [44]. Varios estudios recientes también han demostrado el gran potencial de los algoritmos Structure from Motion para el mapeo y monitoreo de deslizamientos de tierra desde sensores ubicados en UAV [46]-[49]. Se han publicado diferentes estudios que demuestran el uso de datos de UAV para el monitoreo de deslizamientos de tierra [49]-[51]. En un estudio hecho por Niethammer, James, Rothmund, Travelletti y M. Joswiga [52], se evaluó la capacidad del UAV para obtener imágenes de fisuras y desplazamientos en la superficie del deslizamiento de tierra, y la precisión del DSM derivado de imágenes de UAV se comparó con un DSM creado a partir de TLS. [53] utilizaron diversos sistemas de imágenes y vehículos aéreos no tripulados, entre otras plataformas aerotransportadas, para adquirir imágenes de muy alta resolución del deslizamiento de lodo Super-Sauze en la cuenca de Barcelonnette en los Alpes franceses del sur en cinco fechas diferentes.
Según Barbarella y Fiani [5], en el análisis de la evolución temporal de los deslizamientos de tierra y de los peligros hidrogeológicos relacionados, el TLS parece ser una técnica muy adecuada para la descripción morfológica y el análisis de desplazamiento. Para Pirotti, Guarnieri y Vettore [54] y Slob y Hack [55], los TLS tienen la ventaja de proporcionar grandes cantidades de datos a alta resolución en muy poco tiempo, y adicionalmente permiten una descripción precisa y detallada del área escaneada. De hecho, la técnica parece ser muy adecuada para las mediciones de deformación por deslizamientos de tierra, especialmente para terrenos de difícil acceso y pendientes pronunciadas, ya que la realización de múltiples levantamientos permite una rápida adquisición de datos en 3D de la superficie del deslizamiento, en la investigación de Jaboyedoff et al. [56], se puede detallar esta técnica aplicada a los deslizamientos de tierra de laderas. Para la clasificación de los datos provenientes de los escáneres láser en clases de terreno y fuera del terreno, en el estudio de Briese [57], se puede encontrar una descripción detallada de varios algoritmos de filtro desarrollados. A continuación, es necesario convertir los datos de puntos espaciados irregularmente en un DEM (Modelo Digital de Elevación) que se puede generar mediante métodos de interpolación apropiados [58]-[60]. La precisión, fiabilidad de un DEM, y su capacidad para representar fielmente la superficie depende tanto de la morfología del terreno como de la densidad de muestreo y del algoritmo de interpolación [61]. Como manifiestan Fiani y Siani [62] y Abellán, Jaboyedoff, Oppikofer y Vilaplana [63], en sus investigaciones, si el objetivo del estudio es monitorear la deformación del suelo a lo largo del tiempo, se deben comparar dos o más DEM para seguimiento de los desplazamientos de varios puntos del terreno. Por ejemplo, en el trabajo de Ujike y Takagi [64], se pueden comparar directamente los DEM obtenidos a lo largo del tiempo simplemente fijando un número de puntos pertenecientes a objetos particulares visibles en las dos nubes de puntos diferentes. Una ventaja que ofrecen los TLS, y que se evidencian en estudios de Barbarella y Fiani [5], Castagnetti, Bertacchini, Corsini y Rivola [65] y Spreafico et al. [66], es que es una técnica muy eficaz que proporciona gran cantidad de datos en poco tiempo y mejora la interpretación que se puede deducir de los cambios, y deformaciones en un deslizamiento de tierra de laderas. Sin embargo, Martha, Kerle, Jetten, Van Westen y Kumar [67] encontraron que los trabajos con esta técnica consumen bastante tiempo y se obtiene una cobertura espacial pobre, por ende, podría haber una la omisión de la estructura real del terreno a escala detallada en la consecución del Modelo Digital de Superficie (DSM). Westoby, Brasington, Glasser, Hambrey y Reynolds [68] señalan que los TLS proporcionan nubes de puntos altamente densas y precisas, no obstante, el despliegue de estos estudios puede 
conllevar bastante tiempo y, algunas veces, se tornan difíciles de ejecutar cuando se tiene que bregar con terrenos muy empinados, mientras que con la tecnología LiDAR aerotransportado, el trabajo de campo es más accesible pero muy costoso. Como se recoge en la tesis doctoral de Campos [69], los métodos tradicionales topográficos fueron reemplazados por nuevas tecnologías que involucran sensores remotos emplazados en vehículos aéreos no tripulados, los cuales ofrecieron un alto grado de precisión y resolución que conllevan a producir resultados de calidad, eficiencia en tiempos, reducción de costos en los monitores de laderas [69].

\section{Conclusiones}

Esta investigación bibliográfica ha evidenciado el gran rendimiento de estas nuevas tecnologías aplicadas a proyectos de Ingeniería Civil, tan sensibles como es la determinación de los riegos que se pueden producir en una ladera inestable los efectos geológicos o los constructivos. Consecuencias que se pueden traducir en sobrecostos a la explotación de una carretera o una hidroeléctrica, y posible pérdida de vidas humanas.

El avance tecnológico de drones imponiéndose cada vez el de múltirotores o alas rotatorias permiten aumentar producción de datos, ajustar los costos, los tiempos, reducir la mano de obra y mayor resolución, exactitud, precisión y fiabilidad en las mediciones, mejor imagen de estas y mayores tiempos de autonomía de vuelo. Con relación a otras prestaciones del drone y el sensor remoto, se puede mencionar que han contribuido a la obtención de información en tiempo real, en zonas de difícil acceso y con condiciones climáticas adversas que equipos de topografía convencional no podrían ejecutar fácilmente.

La aplicación de los drones para realizar tareas de monitoreo de taludes o laderas estables o inestables, han mostrado un nuevo camino en el desarrollo de proyectos de Ingeniería Civil que han permitido generar información en tiempo real, predecir o simular riesgos asociados a la construcción de taludes de carreteras o en hidroeléctricas, y principalmente, tomar decisiones con un alto grado de certeza ante posibles eventos catastróficos.

El binomio Drone más Sensores Remotos ha otorgado adelantos científicos en la Fotogrametría Digital, que han contribuido a describir, delimitar, caracterizar y monitorizar superficies y volúmenes de taludes o laderas, otorgando calidad, precisión y exactitud en la información obtenida.

La nueva tecnología de UAV podría proporcionar una solución asequible para la realización de varios trabajos de campo incluso en terrenos con altas pendientes con el propósito de monitorear un deslizamiento de tierra de laderas. La precisión de este método aún está bajo investigación, pero dado el interés de los investiga- dores a nivel mundial en el uso de los UAV en diferentes proyectos científicos puedan mejorar esta variable.

A nivel académico ya se debería pensar en que fueran estas tecnologías (drone más sensor remoto) una herramienta más y parte de una asignatura teóricopráctica en los programas universitarios de Ingeniería Civil, en la misma área de conocimiento de asignaturas relacionadas con la Geomática.

\section{Agradecimientos}

Este artículo es derivado del Proyecto de Iniciación Científica con código PIC-DIS-3226 y vigencia 2019, financiado por la Vicerrectoría de Investigaciones de la Universidad Militar Nueva Granada.

\section{Referencias}

[1] D. A. Flores, C. Saito, J. A. Paredes y F. Trujillano, "Aerial photography for 3D reconstruction in the Peruvian Highlands through a fixed-wing UAV system," 2017 IEEE International Conference on Mechatronics (ICM), pp. 388-392, 2017. https://doi.org/10.1109/ICMECH.2017.7921137.

[2] H. Xiang y L. Tian, "Method for automatic georeferencing aerial remote sensing (RS) images from an unmanned aerial vehicle (UAV) platform," Biosystems Engineering, vol. 108, n. ${ }^{\circ}$ 2, pp. 104-113, febrero 2011. https://doi.org/10.1016/j.bios ystemseng.2010.11.003.

[3] S. L. Gariano y F. Guzzetti, "Landslides in a changing climate," Earth-Science Reviews, vol. 162, pp. 227-252, noviembre 2016.

https://doi.org/10.1016/j.earscirev.2016.08.011.

[4] D. Petley, "Global patterns of loss of life from landslides," Geology, vol. 40, n. ${ }^{\circ}$ 10, pp. 927-930, 2012. https://doi.org/10.1130/G33217.1.

[5] M. Barbarella y M. Fiani, "Monitoring of large landslides by Terrestrial Laser Scanning techniques: field data collection and processing," European Journal of Remote Sensing, vol. 46, n. ${ }^{\circ}$, pàgs. 126-151, febrero 2013. https://doi.org/10.5 721/EuJRS20134608.

[6] C. Zhong, Y. Liu, P. Gao, W. Chen, H. Li, Y. Hou, T. Nuremanguli y H. Ma, "Landslide mapping with remote sensing: challenges and opportunities," International Journal of Remote Sensing, vol. 41, n. ${ }^{\circ} 4$, pp. 1555-1581, 2020. https://doi.org/10.1080/01431161.2019.1672904.

[7] M. Alizadeh, I. Ngah, M. Hashim, B. Pradhan y A. Beiranvand Pour, "A Hybrid Analytic Network Process and Artificial Neural Network (ANPANN) Model for Urban Earthquake Vulnerability Assessment," Remote Sensing, vol. 10, n. ${ }^{\circ}$ 6, p. $976,2018$.

https://doi.org/10.3390/rs10060975. 
[8] J. M. Wempen, "Application of DInSAR for short period monitoring of initial subsidence due to longwall mining in the mountain west United States," International Journal of Mining Science and Technology, vol. 30, n. ${ }^{\circ}$ 1, pp. 33-37, enero 2020.

https://doi.org/10.1016/j.ijmst.2019.12.011.

[9] J. M. Wempen y M. K. McCarter, "Comparison of L-band and X-band differential interferometric synthetic aperture radar for mine subsidence monitoring in Central Utah," International Journal of Mining Science and Technology, vol. 27, n. ${ }^{\circ}$, pp. 159-163, 2017. https://doi.org/10.1016/j.ij mst.2016.11.012.

[10] R. K. Dahal, S. Hasegawa, T. Masuda y M. Yamanaka, "Roadside slope failures in Nepal during torrential rainfall and their mitigation," Disaster mitigation of debris flow, slope failures and landslide, vol. 2, pp. 503-514, 2006.

[11] L. Solari, A. Barra, G. Herrera, S. Bianchini, O. Monserrat, M. Béjar-Pizarro, R. S. M. Crosetto y S. Moretti, "Fast detection of ground motions on vulnerable elements using Sentinel-1 InSAR data," Geomatics, Natural Hazards and Risk, vol. 9, n. ${ }^{\circ} 1$, pp. 152-174, 2018. https://doi.org/10. 1080/19475705.2017.1413013.

[12] M. Koeva, M. Muneza, C. Gevaert, M. Gerke y F. Nex, "Using UAVs for map creation and updating. A case study in Rwanda," Survey Review, vol. 50, n. ${ }^{\circ} 361$, pp. 312-325, 2018.

https://doi.org/10.1080/00396265.2016.1268756.

[13] F. Nex y F. Remondino, "UAV for 3D mapping applications: a review," Applied Geomatics, vol. 6, pp. 1-15, 2014. https://doi.org/10.1007/s125 18-013-0120-х.

[14] E. Natsagdorj, T. Renchin, M. Kappas, B. Tseveen, C. Dari, O. Tsend y U. O. Duger, "An integrated methodology for soil moisture analysis using multispectral data in Mongolia," Geospatial Information Science, vol. 20, n. ${ }^{\circ} 1$, pp. 46-55, 2017. https://doi.org/10.1080/10095020.2 017.1307666.

[15] G. J. Grenzdörffer y F. Niemeyer, "UAV-based BRDF-measurements of agricultural surfaces with PFIFFikus," International Archives of the Photogrammetry, Remote Sensing and Spatial Information Science, vol. 38, n. ${ }^{\circ}$ 1/C22, pp. 229-234, 2011.

[16] E. Pino, "Los drones una herramienta para una agricultura eficiente: un futuro de alta tecnología," Idesia (Arica), vol. 37, n. ${ }^{\circ} 1$, pp. 75-84, marzo 2019. https://doi.org/10.4067/S0718-3429 2019005000402.

[17] V. Puri, A. Nayyar y L. Raja, "Agriculture drones: A modern breakthrough in precision agriculture," Journal of Statistics and Management
Systems, vol. 20, n. ${ }^{\circ} 4$, pp. 507-518, 2017. https://doi.org/10.1080/09720510.2017.1395171.

[18] M. A. Ruiz Estrada y A. Ndoma, "The uses of unmanned aerial vehicles-UAV's- (or drones) in social logistic: Natural disasters response and humanitarian relief aid," Procedia Computer Science, vol. 149, pp. 375-383, 2019. https://doi.org/ 10.1016/j.procs.2019.01.151.

[19] D. Giordan, A. Manconi, F. Remondino y F. Nex, "Use of unmanned aerial vehicles in monitoring application and management of natural hazards," Geomatics, Natural Hazards and Risk, vol. 8, n. ${ }^{\circ}$ 1, pp. 1-4, 2017. https://doi.org/10.1080/19475 705.2017.1315619.

[20] K. Chong e I. Lee, "A UAV based close-range rapid aerial monitoring system for emergency responses," ISPRS - International Archives of the Photogrammetry Remote Sensing and Spatial Information Sciences, vol. XxxvIII-1/C22, n. ${ }^{\circ}$, pp. 247-252, septiembre 2011. https://doi.org/ 10.5194/isprsarchives-XXXVIII-1-C22-247-2011.

[21] Pere Molina et al., "Drones to the Rescue!," Inside GNSS, pp. 36-47, julio 2012. https://insideg nss.com/drones-to-the-rescue/.

[22] P. Zarco-Tejada y J. Berni, "Monitoreo de la vegetación mediante un sensor de imágenes microhiperespectrales a bordo de un vehículo aéreo no tripulado (UAV)," in Proceedings of the EuroCOW 2012, European espacial data research (EuroSDR), Castelldefels, 8-10 de febrero de 2012.

[23] F. Agüera, F. Carvajal y M. Pérez, "Measuring sunflower nitrogen status from an unmanned aerial vehicle-based system and an on the ground device," ISPRS Annals of the Photogrametry Remote Sensing and Spatial Information Sciences, vol. XXXVIII-1/C22, n. ${ }^{\circ}$ 1, pp. 33-37, 2011. https://doi.org/10.5194/isprsarchives-XXXVIII1-C22-33-2011.

[24] A. Lucieer, S. Robinson, D. Turner, S. Harwin y J. Kelcey, "Using a micro-UAV for ultra-high resolution multi-sensor observations of Antarctic moss beds," International Archives of the Photogrammetry, Remote Sensing and Spatial Information Sciences, vol. XXXIX-B1, pp. 429-433, 2012. https://doi.org/10.5194/isprsarchives-XXXIX-B1 -429-2012.

[25] F. G. Costa, J. Ueyama, T. Braun, G. Pessin, F. S. Osório y P. A. Vargas, "The use of unmanned aerial vehicles and wireless sensor network in agricultural applications," in Simposio internacional de geociencias y teledetección del IEEE (IGARSS), Munich, 22-27 de julio de 2012. https://doi.org/10.1109/IGARSS.2012.6352477.

[26] D. N. Concepcion-Toledo, E. Gonzalez-Suarez, R. A. Garcia-Prado y J. E. Mino-Valdes, "Metodología de la investigación: Origen y construc- 
ción de una tesis doctoral," Revista Científica de la UCSA, vol. 6, n. ${ }^{\circ}$ 1, pp. 76-87, abril 2019. https://doi.org/10.18004/ucsa/2409-8752/2019. 006(01)076-087.

[27] R. Prokešová, M. Kardoš y A. Medved'ová, "Landslide dynamics from high-resolution aerial photographs: A case study from the Western Carpathians, Slovakia," Geomorphology, vol. 101, n. ${ }^{\circ}$ 1-2, pp. 90-101, 2010. https://doi.org/10.1016/ j.geomorph.2009.09.033.

[28] A. Lucieer, D. Turner, D. H. King y S. A. Robinson, "Using an unmanned aerial vehicle (UAV) to capture micro-topography of antarctic moss beds," International Journal Applied Eath Observation Geoinformation, vol. 27, parte A, pp. 5362, abril 2014. https://doi.org/10.1016/j.jag.2 013.05.011.

[29] E. J. Ramírez Chávez, A. Cruz García, A. G. Lagunas Pérez y O. E. Carreño Reyes, "Uso de vehículos aéreos no tripulados para la caracterización del paisaje sumergido; Bahía Estacahuite," Ciencia y Mar, vol. XIX, n. ${ }^{\circ}$ 51, pp. 35-40, septiembre-diciembre 2013.

[30] F. Carvajal, F. Agüera y M. Pérez, "Surveying a Landslide in a Road Embankment Using Unmanned Aerial Vehicle Photogrammetry," ISPRS International Archives of the Photogrammetry, Remote Sensing and Spatial Information Sciences, vol. XXXVIII-1/C22, pp. 201-206, septiembre 2011. https://doi.org/10.5194/isprsarchives-XXXVIII-1 -C22-201-2011.

[31] Manfreda et al., "On the use of unmanned aerial systems for environmental monitoring," Remote Sensing, vol. 10, n. ${ }^{\circ}$ 4, p. 641, 2018. https://doi.org/10.3390/rs10040641.

[32] U. Niethammer, S. Rothmund, M. R. James, J. Travelletti y M. Joswig, "Uav-Based Remote Sensing of Landslides," International Archives of Photogrammetry, Remote Sensing and Spatial Information Sciences, vol. XxxviII, parte 5, pp. 496501, 2010.

[33] H. T. Berie e I. Burud, "Application of unmanned aerial vehicles in earth resources monitoring: focus on evaluating potentials for forest monitoring in Ethiopia," European Journal of Remote Sensing, vol. 51, n. ${ }^{\circ}$ 1, pp. 326-335, 2018. https:// doi.org/10.1080/22797254.2018.1432993.

[34] P. Boccardo, F. Chiabrando, F. Dutto, F. G. Tonolo y A. Lingua, "UAV Deployment Exercise for Mapping Purposes: Evaluation of Emergency Response Applications," Sensors, vol. 15, n. ${ }^{\circ} 7$, pp. 15717-15737, 2015. https://doi.org/10.3390 /s150715717.

[35] M. Silvagni, A. Tonoli, E. Zenerino y M. Chiaberge, "Multipurpose UAV for search and rescue operations in mountain avalanche events," Geo- matics, Natural Hazards and Risk, vol. 8, n. ${ }^{\circ} 1$, pp. 18-33, 2017. https://doi.org/10.1080/19475 705.2016.1238852.

[36] R. R. Murphy, E. Steimle, C. Griffin, C. Cullins, M. Hall y K. Pratt, "Cooperative use of unmanned sea surface and micro aerial vehicles at Hurricane Wilma," Journal of Field Robotics, vol. 25, n. ${ }^{\circ} 3$, pp. 164-180, 2008. https://doi.org/10.100 2/rob.20235.

[37] K. S. Pratt, R. Murphy, S. Stover y C. Griffin, "CONOPS and Autonomy Recommendations for VTOL Small Unmanned Aerial System Based on Hurricane Katrina Operations," Journal of Field Robotics, vol. 26, n. ${ }^{\circ} 8$, pp. 636-650, 2009. https://doi.org/10.1002/rob.20304.

[38] T. Y. Chou, M. L. Yeh, Y. Chen e Y. H. Chen, "Disaster monitoring and management by the unmanned aerial vehicle technology," in Simposio ISPRS TC VII - 100 años ISPRS, Viena, 2010.

[39] P. Molina et al., "Searching lost people with uavs: the system and results of the close-search project," in International Archives of the Photogrammetry, Remote Sensing and Spatial Information Sciences, XXII Congress of the International Society for Photogrammetry and Remote Sensing, Melbourne, 25 de agosto a 1 de septiembre de 2012.

[40] M. Baldo, C. Bicocchi, U. Chiocchini, D. Giordan y G. Lollino, "LIDAR monitoring of mass wasting processes: The Radicofani landslide, Province of Siena, Central Italy," Geomorphology, vol. 105, n. ${ }^{\circ} 3$, pp. 193-201, 2009. https://doi.org/1 0.1016/j.geomorph.2008.09.015.

[41] E. Nissen, A. K. Krishnan, J. R. Arrowsmith y S. Saripalli, "Three-dimensional surface displacements and rotations from differencing pre- and post-earthquake LiDAR point clouds," Geophysical Research Letters, vol. 39, n. ${ }^{\circ} 16,2012$. https://doi.org/10.1029/2012GL052460.

[42] I. Colomina y P. Molina.

[43] B. U. Meinen y D. T. Robinson, "Streambank topography: an accuracy assessment of UAV-based and traditional 3D reconstructions," International Journal of Remote Sensing, vol. 41, n. ${ }^{\circ} 1$, pp. 1-18, 2020. https://doi.org/10.1080/014311 61.2019.1597294.

[44] K. Nikolakopoulos et al., "Preliminary results from active landslide monitoring using multidisciplinary surveys," European Journal of Remote Sensing, vol. 50, n. ${ }^{\circ} 1,2017$. https://doi.org/10.1080/ 22797254.2017.1324741.

[45] G. F. Wieczorek y J. B. Snyder, "Monitoring slope movements," in Geological Monitoring, $R$. Young y L. Norby, Edits., Boulder, Colorado, 2009, p. $245-271$.

https://doi.org/10.1130/9780813760322. 
[46] A. Lucieer, S. M. De Jong y D. Turner, "Mapping landslide displacements using Structure from Motion (SfM) and image correlation of multi-temporal UAV photography," Progress in Physical Geography, vol. 38, n. ${ }^{\circ} 1$, pp. 97-116, 2013.

https://doi.org/10.1177/0309133313515293.

[47] K. Nikolakopoulos et al., "Active landslide monitoring using remote sensing data, GPS measurements and cameras on board UAV," Proc. SPIE 9644, Earth Resources and Environmental Remote Sensing/GIS Applications, vol. VI, n. ${ }^{\circ} 96440 \mathrm{E}$, 20 octubre 2015.

https://doi.org/10.1117/12.2195394.

[48] U. Niethammer, S. Rothmund, U. Schwaderer, J. Zeman y M. Joswig, "Open source image-processing tools for low-cost uav-based landslide investigations," International Archives of the Photogrammetry, Remote Sensing and Spatial Information Sciences - ISPRS Archives, vol. 38, n. ${ }^{\circ}$ 1C22, pp. 161-166, 2011. https://doi.org/10.51 94/isprsarchives-XXXVIII-1-C22-161-2011.

[49] D. Turner, A. Lucieer y S. M. De Jong, "Time series analysis of landslide dynamics using an unmanned aerial vehicle (UAV)," Remote Sensing, vol. 7 , n. ${ }^{\circ} 2$, pp. 1736-1757, 2015. https://doi.org/10.3390/rs70201736.

[50] J. Lin, H. Tao, Y. Wang y Z. Huang, "Practical application of unmanned aerial vehicles for mountain hazards survey," in 18th International Conference on Geoinformatics, Beijing, 2010.

https://doi.org/10.1109/GEOINFORMATICS.2 010.5567777 .

[51] J. Y. Rau, J. P. Jhan, C. F. Lo e Y. S. Lin, "Landslide mapping using imagery acquired by a fixed-wing UAV. ISPRS International Archives of the Photogrammetry," International Archives of the Photogrammetry, Remote Sensing and Spatial Information Sciences, vol. XxxvIII, n. ${ }^{\circ}$ 1/C22, pp. 195-200, 2011.

[52] U. Niethammer, M. R. James, S. Rothmund, J. Travelletti y M. Joswiga, "UAV-based remote sensing of the super-sauze landslide: Evaluation and results," Engineering Geology, vol. 128, pp. 2-11, 2012. https://doi.org/10.1016/j.enggeo.2011.0 3.012 .

[53] A. Stumpf, J. P. Malet, N. Kerle, U. Niethammer y S. Rothmund, "Image-based mapping of surface fissures for the investigation of landslide dynamics," Geomorphology, vol. 186, pp. 12-27, 2012. https://doi.org/10.1016/j.geomorph.2012.1 2.010 .

[54] F. Pirotti, A. Guarnieri y A. Vettore, "State of the art of ground and aerial laser scanning technologies for high-resolution topography of the earth surface," European Journal Remote Sensing, vol.
46, n. ${ }^{\circ}$ 1, pp. 66-78, 2013. https://doi.org/10.57 21/EuJRS20134605.

[55] S. Slob y H. R. Hack, "3D terrestrial laser scanning as a new field measurement and monitoring technique," in Engineering geology for infrastructure planning in Europe: a European perspective, R. Hack, A. R. y R. Charlier, Edits., Berlin/Heilderberg, Springer, 2004, pp. 179-189.

[56] M. Jaboyedoff et al., "Use of LIDAR in landslide investigations: a review," Nat Hazads, vol. 61, pp. 5-28, 2012. https://doi.org/10.1007/s11069-0109634-2.

[57] C. Briese, "Extraction of digital terrain models," in Airborne and terrestrial laser scanning, G. Vosselman y H. Maas, Edits., Dunbeath, Whittles, pp. $135-167$.

[58] K. Kraus y N. Pfeifer, "Advanced DTM generation from LIDAR data," ISPRS Archives, vol. XXXIV-3/W4, pp. 23-30, octubre 2001.

[59] G. Vosselman y G. Sithole, "Experimental comparison of filter algorithms for bare-earth extraction from airborne laser scanning point clouds," ISPRS Journal Photogrammetry Remote Sensing, vol. 1, pp. 85-101, 2004.

[60] N. Pfeifer y G. Mandlburger, "LIDAR data filtering and DTM generation," in Topographic laser ranging and scanning. Principles and processing, J. Shan y C. K. Toth, Edits., Boca Raton, Florida: Taylor and Francis, pp. 307-334.

[61] F. J. Aguilar, F. Agüera, M. A. Aguilar y F. Carvajal, "Effects of terrain morphology, sampling density, and interpolation methods on grid DEM accuracy," Photogrammetric Engineering \&3 Remote Sensing, vol. 71, n. ${ }^{\circ}$ 7, pp. 805-816, 2005. https://doi.org/10.14358/PERS.71.7.805.

[62] M. Fiani y N. Siani, "Comparison of terrestrial laser scanners in production of DEMs for Cetara tower," in CIPA 2005 Xx International Symposium, Torino, 2005.

[63] A. Abellán, M. Jaboyedoff, T. Oppikofer y J. M. Vilaplana.

[64] K. Ujike y M. Takagi, "Measurement of landslide displacement by object extraction with ground based portable Lased Scanner," in Proceedings of the 25th Asian Conference on Remote Sensing, Chiangmai, 2004.

[65] C. Castagnetti, E. Bertacchini, A. Corsini y R. Rivola, "A reliable methodology for monitoring unstable slopes: The multi-platform and multi-sensor approach," Proceedings of SPIE, 9245, Earth Resources and Environmental Remote Sensing/ GIS Application, vol. v, n. ${ }^{\circ} 92450 \mathrm{~J}$, octubre 2014. https://doi.org/10.1117/12.2067407.

[66] M. C. Spreafico et al., "Terrestrial Remote Sensing techniques to complement conventional geomechanical surveys for the assessment of landsli- 
de hazard: The San Leo case study (Italy)," European Journal of Remote Sensing, vol. 48, n. ${ }^{\circ}$, pp. 639-660, 2015. https://doi.org/10.5721/Eu JRS20154835.

[67] T. R. Martha, N. Kerle, V. Jetten, C. J. Van Westen y K. V. Kumar, "Landslide volumetric analysis using cartosat-1-derived dems," IEEE Geoscience And Remote Sensing Letter, vol. 7, n. ${ }^{\circ} 3$, pp. 582-586, 2010. https://doi.org/10.1109/LG RS.2010.2041895.

[68] M. J. Westoby, J. Brasington, N. F. Glasser, M. J. Hambrey y J. M. Reynolds, "Structure-from motion photogrammetry: A low-cost, effective tool for geoscience applications," Geomorphology, vol. 179, pp. 300-314, 2012. https://doi.org/10.1016 /j.geomorph.2012.08.021.

[69] J. Campos, "Diseño innovador de sensor para laderas sistema previsor de taludes," P.h.D. thesis, Universidad Nacional Autónoma de México, 2017. 